\title{
A nomogram for predicting pathological complete response in patients with human epidermal growth factor receptor 2 negative breast cancer
}

Xi Jin ${ }^{1,2+}$, Yi-Zhou Jiang ${ }^{1,2^{*}+}$, Sheng Chen ${ }^{1,2+}$, Ke-Da Yu ${ }^{1,2}$, Ding Ma ${ }^{1,2}$, Wei Sun ${ }^{1,2}$, Zhi-Min Shao ${ }^{1,2}$ and Gen-Hong Di ${ }^{1,2^{*}}$

\begin{abstract}
Background: The response to neoadjuvant chemotherapy has been proven to predict long-term clinical benefits for patients. Our research is to construct a nomogram to predict pathological complete response of human epidermal growth factor receptor 2 negative breast cancer patients.

Methods: We enrolled 815 patients who received neoadjuvant chemotherapy from 2003 to 2015 and divided them into a training set and a validation set. Univariate logistic regression was performed to screen for predictors and construct the nomogram; multivariate logistic regression was performed to identify independent predictors.

Results: After performing the univariate logistic regression analysis in the training set, tumor size, hormone receptor status, regimens of neoadjuvant chemotherapy and cycles of neoadjuvant chemotherapy were the final predictors for the construction of the nomogram. The multivariate logistic regression analysis demonstrated that T4 status, hormone receptor status and receiving regimen of paclitaxel and carboplatin were independent predictors of pathological complete response. The area under the receiver operating characteristic curve of the training set and the validation set was 0.779 and 0.701 , respectively.

Conclusions: We constructed and validated a nomogram to predict pathological complete response in human epidermal growth factor receptor 2 negative breast cancer patients. We also identified tumor size, hormone receptor status and paclitaxel and carboplatin regimen as independent predictors of pathological complete response.
\end{abstract}

Keywords: HER2 negative breast cancer, Neoadjuvant chemotherapy, Nomogram, Pathological complete response

\section{Background}

Breast cancer is the most common malignant disease and the second most common cause of cancer death in women [1]. Neoadjuvant chemotherapy has several advantages compared with adjuvant chemotherapy [2]. It increases the rate of breast conservation and offers the opportunity for patients with locally advanced breast cancer to receive surgery. Moreover, sensitivity to

\footnotetext{
* Correspondence: yizhoujiang@fudan.edu.cn; didy@medmail.com.cn ${ }^{\dagger}$ Equal contributors

${ }^{1}$ Department of Breast Surgery, Fudan University Shanghai Cancer Center Shanghai 200032, China

Full list of author information is available at the end of the article
}

different chemotherapy regimens can be assessed, thus helping to make decisions for subsequent treatment.

Pathological complete response (pCR) has been confirmed to predict long-term clinical benefit for patients receiving neoadjuvant chemotherapy and can serve as a dependable endpoint when investigating the efficiency of different treatment regimens [3]. With the application of human epidermal growth factor receptor 2 blockade using neoadjuvant treatments such as trastuzumab, pertuzumab and lapatinib in human epidermal growth factor receptor 2 (HER2) positive patients, the pCR rate of HER2 positive patients is high (16.8-66.2 \%) [4]. However, the pCR rate of HER2 negative patients is relatively 
low (7.0-16.2 \% for hormone receptor positive, HER2 negative patients and $33.6-35.0 \%$ for triple negative patients) $[3,5]$. Thus, predicting the response to neoadjuvant chemotherapy for HER2 negative patients is essential to optimizing the treatment for individual patients.

Anthracyclines used to be the most common chemotherapeutic agents for breast cancer [6]. However, as taxane-based [7] or platinum-based [8, 9] regimens showed their advantages, the use of anthracyclines has been declining in recent years [10]. The potential impact of this change is still unknown.

A nomogram is a simple graphical representation of a prediction model that helps oncologists assess the predictive information of individual patients [11]. Several earlier studies constructed nomograms to illustrate the impact of different variables on pCR probability [12-14], but none of them focused on HER2 negative patients and different neoadjuvant chemotherapy regimens.

Our current study aims to construct and validate a well-fitting nomogram based on multivariate logistic regression to evaluate the impact of different neoadjuvant chemotherapy regimens as well as the impact of several other variables on the pCR rate among HER2 negative patients in a prospective cohort.

\section{Methods}

\section{Patient population}

Relevant clinical data (age, menopausal status, tumor size, nodal status, regimens of chemotherapy and cycles of chemotherapy), core needle biopsy samples and surgical specimens were collected from Fudan University Shanghai Cancer Center between January 1, 2003 and April 31, 2015.

Overall, 1244 patients who were diagnosed with primary breast cancer and who received neoadjuvant chemotherapy followed by standard surgery were enrolled.

Patients with HER2 positive core needle biopsy samples, with metastatic disease, with missing data or with previous endocrine therapy were not eligible for this study. In total, 429 patients who had missing relevant information, who were HER2 positive or who had received neoadjuvant chemotherapy regimens other than cyclophosphamide, epirubicin and 5-fluorouracil, cyclophosphamide, epirubicin and 5-fluorouracil followed by paclitaxel or docetaxel and epirubicin, navelbine and epirubicin or paclitaxel and carboplatin or paclitaxel and cisplatin were excluded from our study.

The remaining 815 patients were randomized into a training set $(N=500$, enrolled in the nomogram construction) or a validation set $(N=315$, enrolled in the nomogram external validation) (Fig. 1).

\section{Pathology and treatment}

Estrogen receptor, progestogen receptor status and HER2 status were determined by immunohistochemical

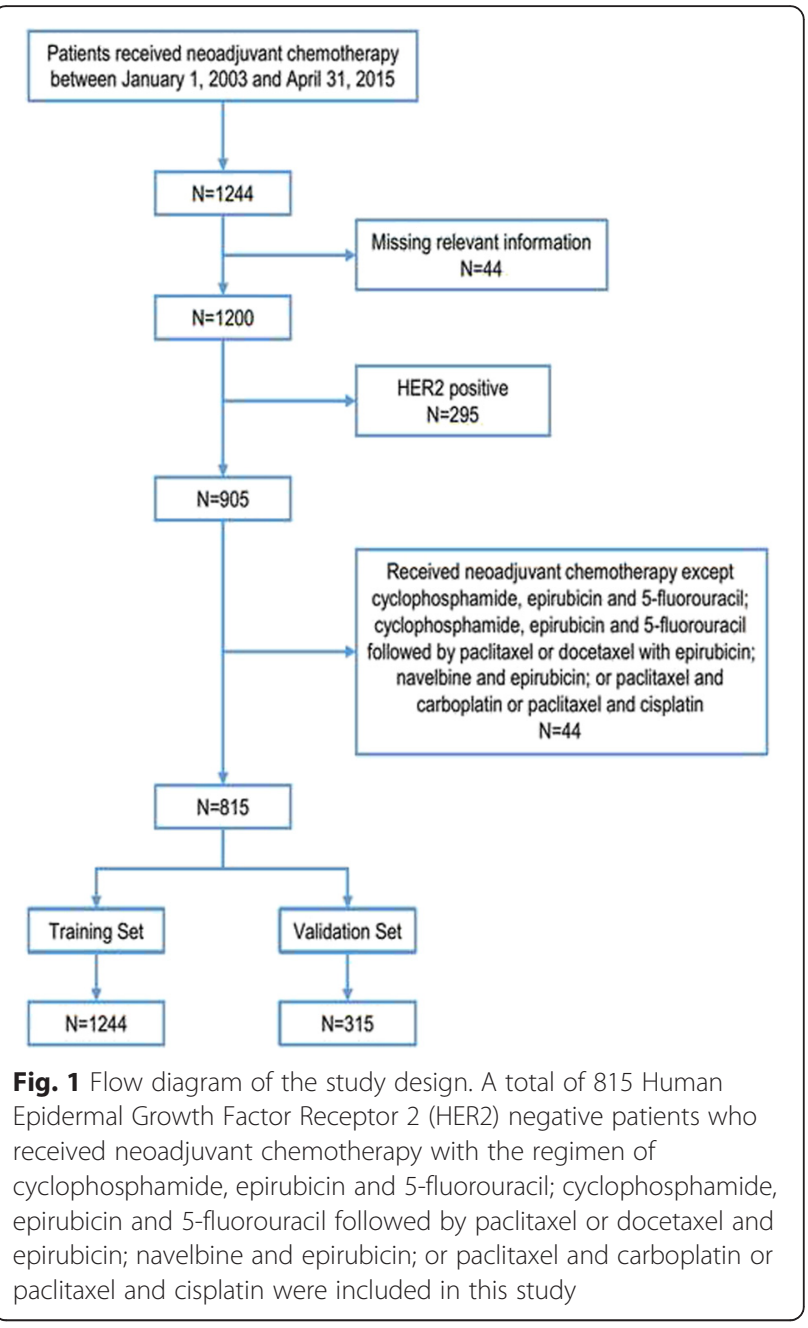

analysis, which was performed with formalin-fixed, paraffin-embedded tissue sections using standard protocols for core needle biopsy specimens by the pathology department of Fudan University Shanghai Cancer Center. The cut-off value for estrogen receptor positivity and progestogen receptor positivity was set at $1 \%$. Absence of both estrogen receptor and progestogen receptor was defined as hormone receptor negative (estrogen receptor negative and progestogen receptor negative); presence of either was defined as hormone receptor positive (estrogen receptor positive or progestogen receptor positive). HER2-positivity was defined as $3(+)$ by immunohistochemical or amplification and was confirmed by fluorescence in situ hybridization. Each specimen was examined independently by two experienced pathologists.

The patients in our cohort received one of the following neoadjuvant chemotherapy regimens for a median of 4 cycles (range, 1-6 cycles): navelbine and epirubicin, cyclophosphamide, epirubicin and 5-fluorouracil, paclitaxel with carboplatin/paclitaxel with cisplatin or epirubicin and 
5-fluorouracil followed by paclitaxel or docetaxel and epirubicin. $\mathrm{pCR}$ was defined as complete disappearance of invasive carcinoma in the breast and regional lymph nodes [3].

\section{Construction of the nomogram}

To develop a well-calibrated and useful nomogram for predicting $\mathrm{PCR}$, possible predictive variables were identified by univariate logistic regression $(P<0.05$ in univariate logistic regression analysis). The Hosmer-Lemeshow test was used to assess the fitness of the nomogram $(P>$ 0.05 indicating good fit) [15]. Multivariate logistic regression analysis was performed to screen independent variables predicting $\mathrm{pCR}$. Odds ratios and $95 \%$ confidence intervals $(\mathrm{CI})$ were calculated.

\section{Evaluating model performance}

The internal validation of our model was performed by a calibration method and the area under the receiver operating characteristic (ROC) curve (AUC). Calibration [16] (visualized as the calibration plot) with a bootstrapping method [17] was used to illustrate the association between the actual probability and the predicted probability. The external validation was achieved by performing the ROC as well as the AUC in a separated population. The AUC ranged from 0 to 1 , with the value of 1 indicating perfect concordance, 0.5 indicating no better than chance, and 0 indicating discordance. Statistical differences between different AUCs were investigated by the DeLong method [18].

\section{Statistical analysis}

Chi-square test was used to evaluate the relationship between neoadjuvant chemotherapy regimens and other characteristics. Fisher's exact test was performed when necessary. All reported $P$-values are two-sided. The statistical analysis was carried out using SPSS (version 20.0; SPSS Company, Chicago, IL) and R software version 3.13 (http://www.r-project.org). The $\mathrm{R}$ package with rms, pROC, Hmisc and ggplot2 (available at URL: http://cran.rproject.org/web/packages/) was used (last accessed on March 9, 2015). All relevant $R$ code were shown in Additional file 1.

\section{Results}

\section{Patient characteristics}

Of the 815 HER 2 negative patients enrolled in this study, 111 (13.6\%) reached pCR (Table 1). Young patients ( $\leq 40$ years) [19] had higher pCR rates than older patients (>40 years) (17.0\% versus $12.8 \%$ ). Pre-menopausal patients $(14.2 \%)$ had higher $\mathrm{pCR}$ rates than those who were post-menopausal (12.8\%). Patients with smaller tumor size and more positive lymph nodes reached $\mathrm{pCR}$ more easily. hormone receptor negative patients $(23.0 \%)$ had higher $\mathrm{pCR}$ rates than hormone receptor positive ones $(9.8 \%)$. Patients who received the paclitaxel with carboplatin/paclitaxel with cisplatin regimen had higher $\mathrm{pCR}$ rates than those who received the cyclophosphamide, epirubicin and 5-fluorouracil, epirubicin and 5fluorouracil followed by paclitaxel or docetaxel and epirubicin or navelbine and epirubicin regimens (19.4\% versus $1.9 \%, 7.8$ and $9.8 \%$, respectively). Patients who received 3 to 4 cycles of neoadjuvant chemotherapy had higher pCR rates $(16.1 \%)$ than other subjects. These results were similar in the training and validation sets.

\section{Predictors for $\mathrm{pCR}$}

In the training set, univariate logistic regression was performed to analyze the association between response to chemotherapy and patient age, menopausal status, tumor size, nodal status, hormone receptor status, regimens of chemotherapy and cycles of chemotherapy (Table 2). Tumor size $(P=0.029)$, hormone receptor status $(<0.001)$, and neoadjuvant chemotherapy regimens $(P<0.001)$ and cycles $(P=0.029)$ were identified to be statistically significant predictors of pCR. No significant differences in $\mathrm{pCR}$ rate were observed among patients with different ages, menopausal statuses or nodal statuses.

Given that the baseline patient characteristics of different neoadjuvant chemotherapy regimens were not in concordance (Additional file 2), we performed multivariate logistic regression analysis to screen for the independent predictors of $\mathrm{pCR}$ (Table 3). Relative to $\mathrm{T} 1$ patients, T4 patients were less likely to achieve pCR $[P=0.015$, odds ratio $=0.281(95 \%$ CI: $0.101-0779)]$. The odds ratio of hormone receptor positive patients was 0.224 (95 \% CI: 0.125-0.400); for hormone receptor negative patients, it was $1(P<0.001)$. After adjustment for tumor size, hormone receptor status and neoadjuvant chemotherapy cycles, those who received paclitaxel with carboplatin/paclitaxel with cisplatin had a statistically significant higher rate of pCR Compared with patients who received cyclophosphamide, epirubicin and 5-fluorouracil $[P=0.003$, odds ratio $=27.696$ (95\% CI: 3.131-245.030)]. Patients who received epirubicin and 5-fluorouracil followed by paclitaxel or docetaxel and epirubicin, navelbine and epirubicin had higher odds ratio than those who received cyclophosphamide, epirubicin and 5-fluorouracil (6.973 and 4.701 versus 1 ), but the difference was not statistically significant. Although we found out the trends that patients receiving only 1-2 cycles neoadjuvant chemotherapy showed lower probability for pCR (odds ratio: 0.579 ) while patients receiving 5-6 cycles neoadjuvant chemotherapy showed higher probability for $\mathrm{pCR}$ (odds ratio: 2.338) than those who received 3-4 cycles of neoadjuvant chemotherapy, different neoadjuvant chemotherapy 
Table 1 Clinicopathologic characteristics of patients

\begin{tabular}{|c|c|c|c|c|c|c|c|c|c|}
\hline & \multicolumn{3}{|l|}{ Overall } & \multicolumn{3}{|c|}{ Training set } & \multicolumn{3}{|c|}{ Validation set } \\
\hline & $\overline{\mathrm{ALL}(\mathrm{N})}$ & $p C R(N)$ & pCR rate & $\overline{\mathrm{ALL}(\mathrm{N})}$ & $\mathrm{pCR}(\mathrm{N})$ & pCR rate & $\overline{\mathrm{ALL}(\mathrm{N})}$ & $p C R(N)$ & $\mathrm{pCR}$ rate \\
\hline Total & 815 & 111 & $13.6 \%$ & 500 & 68 & $13.6 \%$ & 315 & 43 & $13.7 \%$ \\
\hline \multicolumn{10}{|l|}{ Age } \\
\hline$\leq 40$ years & 165 & 28 & $17.0 \%$ & 105 & 17 & $16.2 \%$ & 60 & 11 & $18.3 \%$ \\
\hline$>40$ years & 650 & 83 & $12.8 \%$ & 395 & 51 & $12.9 \%$ & 255 & 32 & $12.5 \%$ \\
\hline \multicolumn{10}{|l|}{ Menopausal status } \\
\hline Pre-menopausal & 457 & 65 & $14.2 \%$ & 276 & 40 & $14.5 \%$ & 181 & 25 & $13.8 \%$ \\
\hline Post-menopausal & 358 & 46 & $12.8 \%$ & 224 & 28 & $12.5 \%$ & 134 & 18 & $13.4 \%$ \\
\hline \multicolumn{10}{|l|}{ Tumor size } \\
\hline $\mathrm{T} 1$ & 89 & 21 & $23.6 \%$ & 60 & 15 & $25.0 \%$ & 29 & 6 & $20.7 \%$ \\
\hline $\mathrm{T} 2$ & 346 & 47 & $13.6 \%$ & 210 & 30 & $14.3 \%$ & 136 & 17 & $12.5 \%$ \\
\hline T3 & 235 & 28 & $11.9 \%$ & 137 & 15 & $10.9 \%$ & 98 & 13 & $13.3 \%$ \\
\hline $\mathrm{T} 4$ & 145 & 15 & $10.3 \%$ & 93 & 8 & $8.6 \%$ & 52 & 7 & $13.5 \%$ \\
\hline \multicolumn{10}{|l|}{ Nodal status } \\
\hline No & 170 & 22 & $12.9 \%$ & 100 & 15 & $15.0 \%$ & 70 & 7 & $10.0 \%$ \\
\hline N1 & 593 & 79 & $13.3 \%$ & 363 & 45 & $12.4 \%$ & 230 & 34 & $14.8 \%$ \\
\hline N2 & 23 & 4 & $17.4 \%$ & 16 & 3 & $18.8 \%$ & 7 & 1 & $14.3 \%$ \\
\hline N3 & 29 & 6 & $20.7 \%$ & 21 & 5 & $23.8 \%$ & 8 & 1 & $12.5 \%$ \\
\hline \multicolumn{10}{|l|}{ Hormone receptor status } \\
\hline Negative & 235 & 54 & $23.0 \%$ & 147 & 36 & $24.5 \%$ & 88 & 18 & $20.5 \%$ \\
\hline Positive & 580 & 57 & $9.8 \%$ & 353 & 32 & $9.1 \%$ & 227 & 25 & $11.0 \%$ \\
\hline \multicolumn{10}{|l|}{ Regimens } \\
\hline $\begin{array}{l}\text { Cyclophosphamide, } \\
\text { epirubicin and } \\
5 \text {-fluorouracil }\end{array}$ & 107 & 2 & $1.9 \%$ & 66 & 1 & $1.5 \%$ & 41 & 1 & $2.4 \%$ \\
\hline $\begin{array}{l}\text { Cyclophosphamide, } \\
\text { epirubicin and 5-fluorouracil } \\
\text { followed by paclitaxel or } \\
\text { docetaxel and epirubicin }\end{array}$ & 116 & 9 & $7.8 \%$ & 73 & 5 & $6.8 \%$ & 43 & 4 & $9.3 \%$ \\
\hline Navelbine and epirubicin & 153 & 15 & $9.8 \%$ & 94 & 8 & $8.5 \%$ & 59 & 7 & $11.9 \%$ \\
\hline $\begin{array}{l}\text { Paclitaxel and carboplatin } \\
\text { or paclitaxel and cisplatin }\end{array}$ & 439 & 85 & $19.4 \%$ & 267 & 54 & $20.2 \%$ & 172 & 31 & $18.0 \%$ \\
\hline \multicolumn{10}{|l|}{ Cycles } \\
\hline $1-2$ & 97 & 3 & $3.1 \%$ & 61 & 2 & $3.3 \%$ & 36 & 1 & $2.8 \%$ \\
\hline $3-4$ & 578 & 93 & $16.1 \%$ & 359 & 58 & $16.2 \%$ & 219 & 35 & $16.0 \%$ \\
\hline $5-6$ & 140 & 15 & $10.7 \%$ & 80 & 8 & $10.0 \%$ & 60 & 7 & $11.7 \%$ \\
\hline
\end{tabular}

Abbreviations: $p C R$ pathological complete response

cycles were not statistically significant for predicting pCR.

We performed logistic regression to explore the predictors for $\mathrm{pCR}$ separately both in hormone receptor positive and negative cohort. Tumor status (T3 vs T1, T4 vs T1) was only statistically significant in hormone receptor positive patients and not in hormone receptor negative patients. Nodal status was not statistically significant in either group. Epirubicin and 5-fluorouracil followed by paclitaxel or docetaxel with epirubicin and navelbine with epirubicin showed statistically significant superiority to cyclophosphamide, epirubicin and 5fluorouracil regimens in hormone receptor negative patients, but not in hormone receptor positive patients, while paclitaxel with carboplatin/paclitaxel with cisplatin regimen treated patients had statistically significant higher $\mathrm{pCR}$ in overall patients. Only hormone receptor negative patients who received 1-2 cycles had statistically significant lower $\mathrm{pCR}$ rate than those receiving 3-4 cycles (Additional file 3). In addition, we found that 
Table 2 Univariate logistic regression analysis of different variables predicting $\mathrm{PCR}$ in the training set

\begin{tabular}{|c|c|c|c|}
\hline & $P$ & OR & $95 \% \mathrm{Cl}$ \\
\hline \multicolumn{4}{|l|}{ Total } \\
\hline Age & 0.385 & & \\
\hline$\leq 40$ years & & 1 & \\
\hline$>40$ years & 0.385 & 0.767 & $0.423-1.394$ \\
\hline Menopausal status & 0.518 & & \\
\hline Pre-menopausal & & 1 & \\
\hline Post-menopausal & 0.518 & 0.843 & $0.502-1.416$ \\
\hline Tumor Size & 0.029 & & \\
\hline $\mathrm{T} 1$ & & 1 & \\
\hline $\mathrm{T} 2$ & 0.052 & 0.500 & $0.248-1.007$ \\
\hline T3 & 0.014 & 0.369 & $0.167-0.815$ \\
\hline T4 & 0.008 & 0.282 & $0.111-0.716$ \\
\hline Nodal status & 0.432 & & \\
\hline No & & 1 & \\
\hline N1 & 0.493 & 0.802 & $0.426-1.508$ \\
\hline N2 & 0.701 & 1.308 & $0.332-5.147$ \\
\hline N3 & 0.328 & 1.171 & $0.564-5.561$ \\
\hline Hormone receptor status & $<0.001$ & & \\
\hline Negative & & 1 & \\
\hline Positive & $<0.001$ & 0.307 & $0.182-0.518$ \\
\hline Regimens & $<0.001$ & & \\
\hline $\begin{array}{l}\text { Cyclophosphamide, epirubicin } \\
\text { and } 5 \text {-fluorouracil }\end{array}$ & & 1 & \\
\hline $\begin{array}{l}\text { Cyclophosphamide, epirubicin } \\
\text { and 5-fluorouracil followed by } \\
\text { paclitaxel or docetaxel and } \\
\text { epirubicin }\end{array}$ & 0.158 & 4.779 & $0.544-42.018$ \\
\hline Navelbine and epirubicin & 0.094 & 6.047 & $0.738-49.558$ \\
\hline $\begin{array}{l}\text { Paclitaxel and carboplatin } \\
\text { or paclitaxel and cisplatin }\end{array}$ & 0.006 & 16.479 & $2.236-121.451$ \\
\hline Cycles & 0.029 & & \\
\hline $3-4$ & & 1 & \\
\hline $1-2$ & 0.018 & 0.176 & $0.042-0.740$ \\
\hline $5-6$ & 0.143 & 0.577 & $0.264-1.261$ \\
\hline
\end{tabular}

Abbreviations: $p C R$ pathological complete response, $O R$ odds ratio, $C l$ confidence interval

among paclitaxel with carboplatin/paclitaxel with cisplatin treated patients, hormone receptor negative (triple negative) patients had higher rate of pCR rate $(38.9 \%$, Chi-square test $P<0.001)$ than hormone receptor positive patients (13.0\%) (Additional file 4).

\section{Construction and validation of the nomogram}

Statistically significant predictors in univariate logistic regression analysis (tumor size, hormone receptor status, neoadjuvant chemotherapy regimens and cycles) were included into the nomogram construction (Fig. 2). The
Table 3 Multivariable logistic regression analysis of possible variables ( $P<0.05$ in univariate logistic regression analysis) predicting $\mathrm{PCR}$

\begin{tabular}{|c|c|c|c|}
\hline & $\mathrm{P}$ & OR & $95 \% \mathrm{Cl}$ \\
\hline \multicolumn{4}{|l|}{ Tumor size } \\
\hline $\mathrm{T} 1$ & & 1 & \\
\hline $\mathrm{T} 2$ & 0.186 & 0.576 & $0.255-1.304$ \\
\hline T3 & 0.544 & 0.737 & $0.275-1.975$ \\
\hline $\mathrm{T} 4$ & 0.015 & 0.281 & $0.101-0.779$ \\
\hline \multicolumn{4}{|l|}{ Hormone receptor status } \\
\hline Negative & & 1 & \\
\hline Positive & $<0.001$ & 0.224 & $0.125-0.400$ \\
\hline \multicolumn{4}{|l|}{ Regimens } \\
\hline $\begin{array}{l}\text { Cyclophosphamide, epirubicin } \\
\text { and } 5 \text {-fluorouracil }\end{array}$ & & 1 & \\
\hline $\begin{array}{l}\text { Cyclophosphamide, epirubicin } \\
\text { and } 5 \text {-fluorouracil followed by } \\
\text { paclitaxel or docetaxel and } \\
\text { epirubicin }\end{array}$ & 0.208 & 4.673 & $0.423-51.590$ \\
\hline Navelbine and epirubicin & 0.078 & 6.999 & $0.804-60.897$ \\
\hline $\begin{array}{l}\text { Paclitaxel and carboplatin or } \\
\text { paclitaxel and cisplatin }\end{array}$ & 0.003 & 27.696 & $3.131-245.030$ \\
\hline \multicolumn{4}{|l|}{ Cycles } \\
\hline $3-4$ & & 1 & \\
\hline $1-2$ & 0.500 & 0.579 & $0.118-2.834$ \\
\hline $5-6$ & 0.217 & 2.338 & $0.606-9.017$ \\
\hline
\end{tabular}

Abbreviations: $p C R$ pathological complete response, $O R$ odds ratio, $C I$ confidence interval

total points were added up by the points of each variable (top scale). The pCR probability depended on the total points (bottom scale). The $P$-value for the HosmerLemeshow test was 0.817 , indicating good fit of the model.

The calibration of the nomogram was performed internally by a calibration plot with bootstrap sampling $(n=1000)$ (Fig. 3). The calibration plot illustrated that the nomogram was well calibrated.

Next, we constructed the ROC to further validate the nomogram internally in the training set (Fig. 4a) and externally in the validation set (Fig. 4b). In the training set, the AUC was 0.779 (95\% CI: 0.718-0.839). In the validation set, the AUC was slightly lower: 0.703 (95\% CI: 0.624-0.782). The difference between two AUCs was not statistical significant $(P=0.132)$. These results illustrated that the predicted and observed $\mathrm{PCR}$ probabilities were concordant.

\section{Nomogram performance in individual patients}

To display the application of the nomogram, we took two breast cancer patients who had received neoadjuvant chemotherapy as examples. The first patient was to receive epirubicin and 5-fluorouracil followed by paclitaxel 


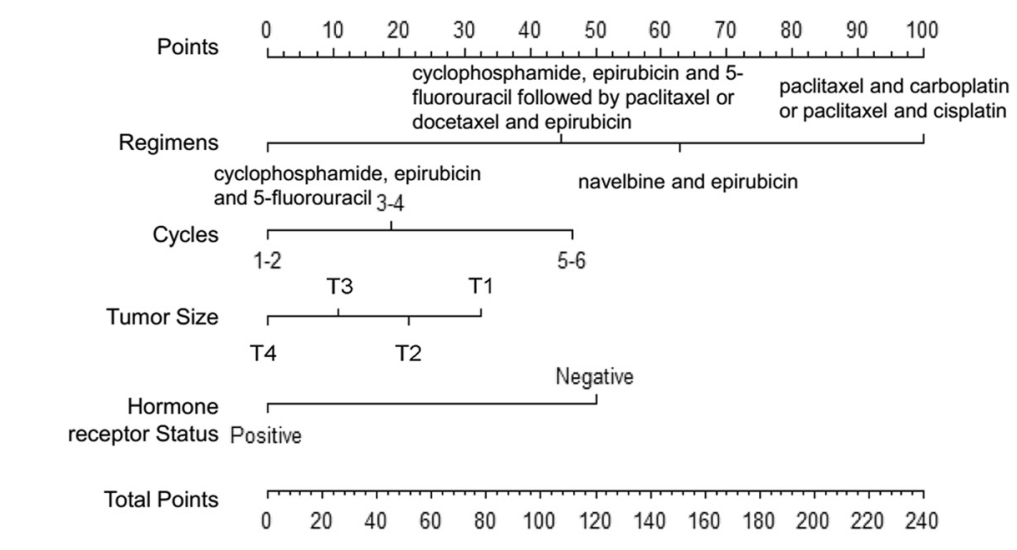

pCR Probability (\%)

$\begin{array}{lllllllll}0.01 & 0.05 & 0.1 & 0.2 & 0.3 & 0.4 & 0.5 & 0.6 & 0.7\end{array}$

Fig. 2 Nomogram predicting the probability of pathological complete response (pCR) after neoadjuvant chemotherapywith the regimen of cyclophosphamide, epirubicin and 5-fluorouracil; cyclophosphamide, epirubicin and 5-fluorouracil followed by paclitaxel or docetaxel and epirubicin; navelbine and epirubicin; or paclitaxel and carboplatin or paclitaxel and cisplatin

or docetaxel and epirubicin as an neoadjuvant chemotherapy regimen (45 points) for four cycles (19 points); his tumor size was T2 (22 points) and his hormone receptor status was positive (0 points). According to the nomogram, his probability of reaching $\mathrm{pCR}$ was approximately 0.01 to 0.05 (total points: 86). The second patient was to receive paclitaxel with carboplatin/paclitaxel with cisplatin as an neoadjuvant chemotherapy regimen (100 points) for four cycles (19 points); his tumor size was T4 (0 points) and his hormone receptor status was negative (50 points). According to the nomogram, his probability of reaching pCR was approximately 0.2 to 0.3 (total points: 169). As a result of using this nomogram, clinicians can obtain an

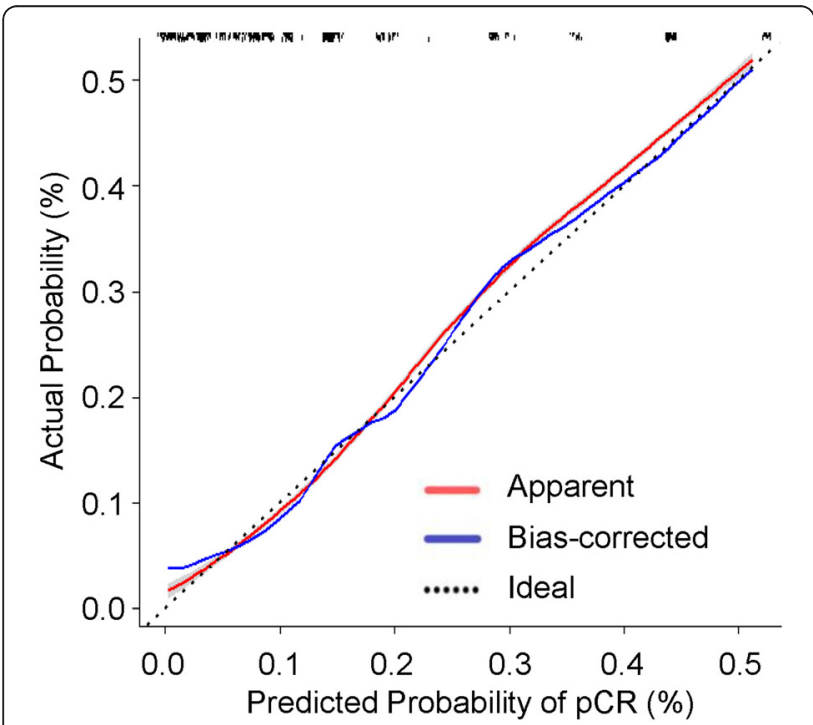

Fig. 3 Calibration plot of the nomogram for the probability of pathological complete response (pCR) (bootstrap 1000 repetitions) overview of the response of different treatments for individual patients.

\section{Discussion}

Based on the logistic regression, we screened for predictors and constructed a concise and well fitted nomogram containing the variables of tumor size, hormone receptor status, regimens of neoadjuvant chemotherapy and cycles of neoadjuvant chemotherapy to predict the $\mathrm{pCR}$ rate of HER2 negative patients. This would be a convenient application for clinicians. Using the method of calibration plot with bootstrap sampling, as well as internal and external validation by AUC and ROC, the nomogram proved to be of good fitness.

In this study, we first screened variables that could predict the response to neoadjuvant chemotherapy by univariate logistic regression. Tumor size, hormone receptor status, and neoadjuvant chemotherapy regimens and cycles were included in the construction of the nomogram. Next, we intended to identify several independent predictors of the $\mathrm{pCR}$ rate. In the multivariate logistic regression analysis, we found that T4 status $(P=$ 0.015, odds ratio: $0.281,95 \% \mathrm{CI}: 0.101-0.779)$, hormone receptor positivity $(P<0.001$, odds ratio: $0.224,95 \% \mathrm{CI}$ : $0.125-0.400)$ and receiving the paclitaxel with carboplatin/paclitaxel with cisplatin regimen $(P=0.003$, odds ratio: 27.696, $95 \%$ CI: 3.131-245.030) were the most important predictors of $\mathrm{pCR}$ in this model. Compared with $\mathrm{T} 1$ patients, $\mathrm{T} 4$ patients had worse responses to chemotherapy, which is consistent with previous research [20]. Hormone receptor status was another independent predictor, and hormone receptor positive patients had lower $\mathrm{pCR}$ rates than hormone receptor negative patients. Our findings are concordant with 

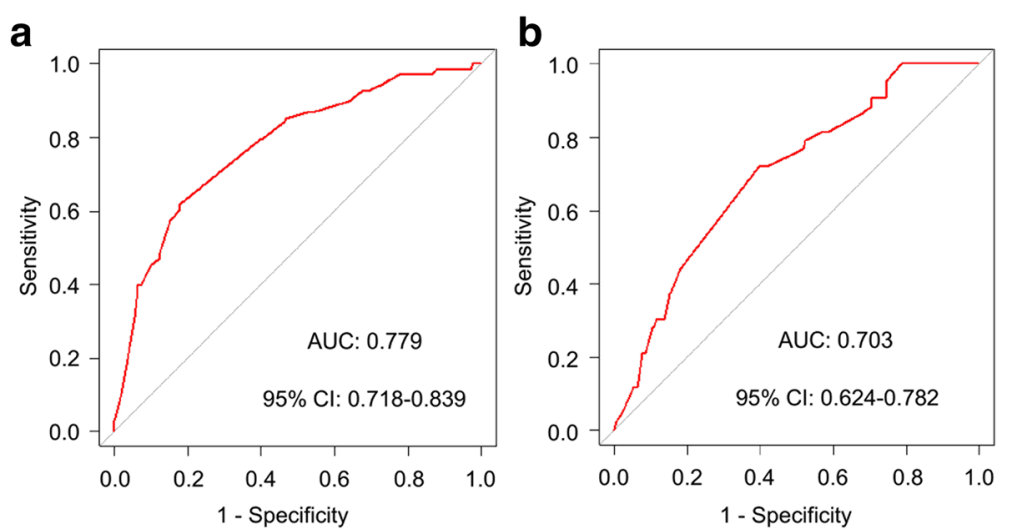

Fig. 4 Validation of the Nomogram. a Internal validation using receiver operating characteristic (ROC) curve. The area under the ROC curve (AUC) is $0.779,95 \%$ confidence intervals (Cl): 0.718-0.839. b External validation using ROC. The AUC is $0.703,95 \%$ Cl: $0.622-0.780$

previous studies [20-22] that show that hormone receptor positive tumor cells are less sensitive to chemotherapy compared with hormone receptor negative cells. Patients treated with paclitaxel with carboplatin/paclitaxel with cisplatin had better neoadjuvant chemotherapy responses compared with those treated with cyclophosphamide, epirubicin and 5-fluorouracil. Anthracyclines such as epirubicin and doxorubicin were once considered to be the most effective agents in the treatment of breast cancer, but the use of them has been declining recently [10]. In our current study, the anthracycline-based regimens included cyclophosphamide, epirubicin and 5-fluorouracil, epirubicin and 5-fluorouracil followed by paclitaxel or docetaxel with epirubicin and navelbine with epirubicin. Cyclophosphamide, epirubicin and 5-fluorouracil was the standard anthracycline-based regimen, and the pCR rate after 6 cycles of cyclophosphamide, epirubicin and 5fluorouracil was reported to be 14-15 \% [23, 24]. However, only $1.9 \%$ of patients who received cyclophosphamide, epirubicin and 5-fluorouracil in our study reached $\mathrm{pCR}$, which may be partially due to the relatively higher proportion of larger tumor size (T3: $50.5 \%$; T4: $13.1 \%)$ and fewer neoadjuvant chemotherapy cycles received (1-2 cycles: $49.5 \%$ ) in the cyclophosphamide, epirubicin and 5-fluorouracil cohort. The total pCR rate for epirubicin and 5-fluorouracil followed by paclitaxel or docetaxel and epirubicin patients was low (7.8 \%) which may due to the relatively higher proportion of hormone receptor positive patients $(87.1 \%)$. The cumulative cardiac toxicity of anthracyclines has also limited its use, especially in older patients or in those with cardiovascular comorbidities. Therefore, non-anthracycline based regimens are required. Paclitaxel, a mitotic inhibitor and anti-microtubule agent, results in a G2-M phase arrest [25]. Carboplatin and cisplatin share similar anti-cancer mechanisms, as they are both DNA alkylating agents [26]. The combination of paclitaxel and platinum is now widely used in breast cancer patients, and the agents have no overlapping toxicities [27]. Previous research has already assessed the efficacy and the toxicity of the paclitaxel with carboplatin/ paclitaxel with cisplatin regimen in adjuvant therapy and in neoadjuvant chemotherapy. The $\mathrm{pCR}$ rate of patients who received paclitaxel with carboplatin/paclitaxel with cisplatin as neoadjuvant chemotherapy ranged from 9.5 to $19.4 \%[28,29]$. The data from our center is $19.4 \%$, similar to previous studies. The paclitaxel with carboplatin/paclitaxel with cisplatin regimen achieved greater therapeutic effect than any anthracycline-based regimens, especially in triple negative breast cancer patients. Triple negative breast cancer patients have higher rate of BRCA1/2 (Breast Cancer 1/2) mutation and are sensitive to platinum (because of the deficiencies in the DNA repair mechanism) [30, 31]. In aggregate, these results suggested that platinum contained therapy is recommend for triple negative breast cancer patients.

The nomogram provides a simple graphical representation of sophisticated statistical prediction models and has been accepted as a reliable tool for predicting clinical events. It is especially widely used in oncology [11]. Previously, several studies constructed nomograms to predict the $\mathrm{pCR}$ rate of neoadjuvant chemotherapy. The first of these studies appeared in 2005 [12]. Rouzier et al. constructed two nomograms to predict the responses to anthracycline-based neoadjuvant chemotherapy and to combined anthracycline and paclitaxel neoadjuvant chemotherapy. The nomograms were validated externally. Colleoni et al. constructed a nomogram to predict pCR probability based on a population of 783 patients [13]. The nomogram proved to be well fitted after external validation by 101 patients. However, the HER2 status was not mentioned in these two studies. Keam et al. constructed another nomogram to predict $\mathrm{pCR}$ and predict which patients would not relapse [14]. Overall, 370 patients who received 3 cycles of neoadjuvant docetaxel 
or doxorubicin were included in this study. However, the HER2 status was not stratified and the validation of the nomogram was only performed internally. The advantage of our research is that we first constructed a nomogram for predicting the $\mathrm{pCR}$ rate among HER2 negative patients, and the nomogram was proven to be well fitted by internal and external validation. We selected HER2 negative patients as our target population for two reasons. First, the pCR rates of these patients were relatively low, so individualized therapy for each patient was required. Second, confounding variables such as HER2 blockade treatment were limited in our cohort. Additionally, we discovered that paclitaxel with carboplatin/ paclitaxel with cisplatin was the more favored neoadjuvant chemotherapy regimen compared with cyclophosphamide, epirubicin and 5-fluorouracil in HER2 negative patients.

One limitation of our study was that the design was a single center analysis. Applying the nomogram in another database will greatly improve the power of our current result, and we have carefully searched through existing public databases. Unfortunately, we were unable to find a proper database containing all of the variables analyzed in our current study (age, menopause status, tumor size, nodal status, hormone receptor status, neoadjuvant chemotherapy regimens, neoadjuvant chemotherapy cycles and response to neoadjuvant chemotherapy). We expect to assess the nomogram with large-scale randomized prospective clinical trials. The efficacy and safety of the paclitaxel with carboplatin/paclitaxel with cisplatin regimen used in neoadjuvant chemotherapy also needs to be assessed. Another limitation was that the molecular mechanisms of the paclitaxel with carboplatin/paclitaxel with cisplatin regimen (more so than the cyclophosphamide, epirubicin and 5-fluorouracil regimen) were unclear so further research is required in the future to study these mechanisms.

\section{Conclusion}

Our current study screened for several predictors and constructed a well fitted nomogram based on those predictors to predict the pCR rate among HER2 negative breast cancer patients.

\section{Additional files}

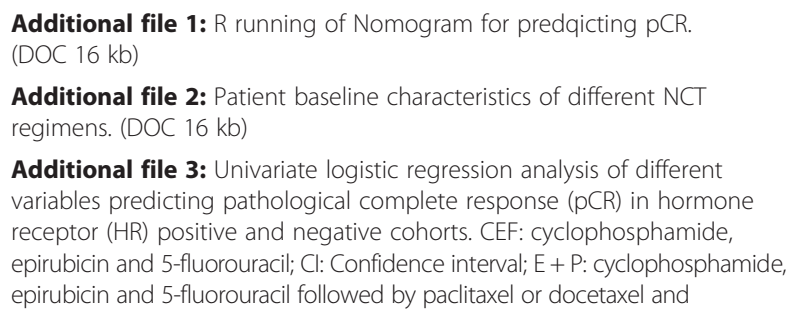

Additional file 1: $R$ running of Nomogram for predqicting $p C R$. (DOC $16 \mathrm{~kb}$ )

Additional file 2: Patient baseline characteristics of different NCT regimens. (DOC $16 \mathrm{~kb}$ )

Additional file 3: Univariate logistic regression analysis of different variables predicting pathological complete response ( $p C R)$ in hormone receptor (HR) positive and negative cohorts. CEF: cyclophosphamide, epirubicin and 5-fluorouracil; Cl: Confidence interval; E + P: cyclophosphamide, epirubicin and 5-fluorouracil followed by paclitaxel or docetaxel and

epirubicin; NE: navelbine and epirubicin; OR: odds ratios; PC: paclitaxel and carboplatin or paclitaxel and cisplatin. (DOC $1415 \mathrm{~kb}$ )

Additional file 4: Pathological complete response $(\mathrm{pCR})$ of different neoadjuvant chemotherapy (NCT) regimens in hormone receptor (HR) positive and negative cohorts. (DOC $152 \mathrm{~kb})$

\section{Abbreviations}

$A U C$, the area under the ROC curve; $\mathrm{Cl}$, confidence interval; HER2, human epidermal growth factor receptor 2; pCR, pathological complete response; ROC, receiver operating characteristic

\section{Acknowledgements}

The authors are grateful to Jiong Wu, Guang-Yu Liu and Zhen-Zhou Shen for their excellent data management.

\section{Funding}

This work was supported by grants from the Research Project of Fudan University Shanghai Cancer Center (YJ201401); the National Natural Science Foundation of China (81572583, 81502278, 81372848, 81370075); the Municipal Project for Developing Emerging and Frontier Technology in Shanghai Hospitals (SHDC12010116); the Cooperation Project of Conquering Major Diseases in Shanghai Municipality Health System (2013ZYJB0302); the Innovation Team of the Ministry of Education (IRT1223); and the Shanghai Key Laboratory of Breast Cancer (12DZ2260100). The funders had no role in the study design, data collection and analysis, decision to publish, or preparation of the manuscript.

\section{Availability of data and materials}

The dataset supporting the conclusions of this article is included within the article.

\section{Authors' contributions}

JX and YZJ contributed to the conception of the study, data analysis and interpretation, and writing the manuscript. JX, SW and MD helped in the nomogram construction. CS made tissue sections and participated in immunohistochemical analysis. YKD, DGH and SZM contributed to the collection and assembly of data. All authors read and approved the final manuscript.

\section{Competing interests}

The authors declare that they have no competing interests.

\section{Consent for publication}

Not applicable.

\section{Ethics approval and consent to participate}

All the procedures followed were in accordance with the Helsinki Declaration (1964, amended in 1975, 1983, 1989, 1996 and 2000) of the World Medical Association. This study was approved by the Ethics Committee of Fudan University shanghai Cancer Center, and each participant signed an informed consent document.

\section{Author details}

'Department of Breast Surgery, Fudan University Shanghai Cancer Center, Shanghai 200032, China. ${ }^{2}$ Department of Oncology, Shanghai Medical College, Fudan University, Shanghai 200032, China.

Received: 14 April 2016 Accepted: 29 July 2016

Published online: 05 August 2016

\section{References}

1. DeSantis C, Siegel R, Bandi P, Jemal A. Breast cancer statistics, 2011. CA Cancer J Clin. 2011;61(6):409-18.

2. Generali D, Ardine M, Strina C, Milani M, Cappelletti MR, Zanotti L, Forti M, Bedussi F, Martinotti M, Amoroso V, et al. Neoadjuvant treatment approach: the Rosetta stone for breast cancer? J Natl Cancer Inst Monogr. 2015;2015(51):32-5.

3. Cortazar P, Zhang L, Untch M, Mehta K, Costantino JP, Wolmark N, Bonnefoi H, Cameron D, Gianni L, Valagussa P, et al. Pathological complete response 
and long-term clinical benefit in breast cancer: the CTNeoBC pooled analysis. Lancet. 2014;384(9938):164-72.

4. Zardavas D, Piccart M. Neoadjuvant therapy for breast cancer. Annu Rev Med. 2015;66:31-48

5. Straver ME, Rutgers EJ, Rodenhuis S, Linn SC, Loo CE, Wesseling J, Russel NS, Oldenburg HS, Antonini N, Vrancken Peeters MT. The relevance of breast cancer subtypes in the outcome of neoadjuvant chemotherapy. Ann Surg Oncol. 2010;17(9):2411-8.

6. Harlan LC, Clegg LX, Abrams J, Stevens JL, Ballard-Barbash R. Communitybased use of chemotherapy and hormonal therapy for early-stage breast cancer: 1987-2000. J Clin Oncol. 2006;24(6):872-7.

7. Jones SE, Savin MA, Holmes FA, O'Shaughnessy JA, Blum JL, Vukelja S, Mclntyre KJ, Pippen JE, Bordelon JH, Kirby R, et al. Phase III trial comparing doxorubicin plus cyclophosphamide with docetaxel plus cyclophosphamide as adjuvant therapy for operable breast cancer. J Clin Oncol. 2006;24(34):5381-7.

8. Silver DP, Richardson AL, Eklund AC, Wang ZC, Szallasi Z, Li Q, Juul N, Leong CO, Calogrias D, Buraimoh A, et al. Efficacy of neoadjuvant Cisplatin in triple-negative breast cancer. J Clin Oncol. 2010;28(7):1145-53.

9. von Minckwitz G, Schneeweiss A, Loibl S, Salat C, Denkert C, Rezai M, Blohmer JU, Jackisch C, Paepke S, Gerber B, et al. Neoadjuvant carboplatin in patients with triple-negative and HER2-positive early breast cancer (GeparSixto; GBG 66): a randomised phase 2 trial. Lancet Oncol. 2014;15(7):747-56

10. Giordano SH, Lin YL, Kuo YF, Hortobagyi GN, Goodwin JS. Decline in the use of anthracyclines for breast cancer. J Clin Oncol. 2012;30(18):2232-9.

11. lasonos A, Schrag D, Raj GV, Panageas KS. How to build and interpret a nomogram for cancer prognosis. J Clin Oncol. 2008;26(8):1364-70.

12. Rouzier R, Pusztai L, Delaloge S, Gonzalez-Angulo AM, Andre F, Hess KR, Buzdar AU, Garbay JR, Spielmann M, Mathieu MC, et al. Nomograms to predict pathologic complete response and metastasis-free survival after preoperative chemotherapy for breast cancer. J Clin Oncol. 2005;23(33):8331-9.

13. Colleoni M, Bagnardi V, Rotmensz N, Viale G, Mastropasqua M, Veronesi P, Cardillo A, Torrisi R, Luini A, Goldhirsch A. A nomogram based on the expression of Ki-67, steroid hormone receptors status and number of chemotherapy courses to predict pathological complete remission after preoperative chemotherapy for breast cancer. Eur J Cancer. 2010;46(12):2216-24.

14. Keam B, Im SA, Park S, Nam BH, Han SW, Oh DY, Kim JH, Lee SH, Han W Kim DW, et al. Nomogram predicting clinical outcomes in breast cancer patients treated with neoadjuvant chemotherapy. J Cancer Res Clin Oncol. 2011;137(9):1301-8.

15. Hosmer DW, Lemeshow S. Assessing the fit of the model. In: Applied logistic regression. Wiley; 2005: 143-202. doi: 10.1002/0471722146.

16. Harrell Jr FE, Lee KL, Mark DB. Multivariable prognostic models: issues in developing models, evaluating assumptions and adequacy, and measuring and reducing errors. Stat Med. 1996;15(4):361-87.

17. Steyerberg EW, Harrell Jr FE, Borsboom GJ, Eijkemans MJ, Vergouwe Y, Habbema JD. Internal validation of predictive models: efficiency of some procedures for logistic regression analysis. J Clin Epidemiol. 2001;54(8):774-81.

18. DeLong ER, DeLong DM, Clarke-Pearson DL. Comparing the areas under two or more correlated receiver operating characteristic curves: a nonparametric approach. Biometrics. 1988;44(3):837-45.

19. Collins LC, Marotti JD, Gelber S, Cole K, Ruddy K, Kereakoglow S, Brachtel EF, Schapira L, Come SE, Winer EP, et al. Pathologic features and molecular phenotype by patient age in a large cohort of young women with breast cancer. Breast Cancer Res Treat. 2012;131(3):1061-6.

20. Caudle AS, Gonzalez-Angulo AM, Hunt KK, Liu P, Pusztai L, Symmans WF, Kuerer HM, Mittendorf EA, Hortobagyi GN, Meric-Bernstam F. Predictors of tumor progression during neoadjuvant chemotherapy in breast cancer. J Clin Oncol. 2010;28(11):1821-8.

21. Precht LM, Lowe KA, Atwood M, Beatty JD. Neoadjuvant chemotherapy of breast cancer: tumor markers as predictors of pathologic response, recurrence, and survival. Breast J. 2010;16(4):362-8.

22. Tan MC, Al Mushawah F, Gao F, Aft RL, Gillanders WE, Eberlein TJ, Margenthaler JA. Predictors of complete pathological response after neoadjuvant systemic therapy for breast cancer. Am J Surg. 2009;198(4):520-5

23. Mouret-Reynier M-A, Abrial CJ, Ferrière J-P, Amat S, Curé HD, Kwiatkowski FG, Feillel VA, Lebouëdec G, Penault-Llorca FM, Chollet PJM. Neoadjuvant
FEC 100 for operable breast cancer: eight-year experience at centre jean Perrin. Clin Breast Cancer. 2004:5(4):303-7.

24. Buzdar AU. Preoperative chemotherapy treatment of breast cancer-a review. Cancer. 2007;110(11):2394-407.

25. Jiang YZ, Yu KD, Peng WT, Di GH, Wu J, Liu GY, Shao ZM. Enriched variations in TEKT4 and breast cancer resistance to paclitaxel. Nat Commun. 2014;5:3802.

26. Xiong X, Sui M, Fan W, Kraft AS. Cell-cycle dependent antagonistic interactions between paclitaxel and carboplatin in combination therapy. Cancer Biol Ther. 2014;6(7):1067-73.

27. Pentheroudakis G, Razis E, Athanassiadis A, Pavlidis N, Fountzilas G. Paclitaxel-carboplatin combination chemotherapy in advanced breast cancer: accumulating evidence for synergy, efficacy, and safety. Med Oncol. 2006;23(2):147-60

28. Chen XS, Nie XQ, Chen CM, Wu JY, Wu J, Lu JS, Shao ZM, Shen ZZ, Shen $\mathrm{KW}$. Weekly paclitaxel plus carboplatin is an effective nonanthracyclinecontaining regimen as neoadjuvant chemotherapy for breast cancer. Ann Oncol. 2010;21(5):961-7.

29. Gogas H, Pectasides D, Kostopoulos I, Lianos E, Skarlos D, Papaxoinis G, Bobos M, Kalofonos HP, Petraki K, Pavlakis K, et al. Paclitaxel and carboplatin as neoadjuvant chemotherapy in patients with locally advanced breast cancer: a phase II trial of the Hellenic cooperative oncology group. Clin Breast Cancer. 2010;10(3):230-7

30. Hurley J, Reis IM, Rodgers SE, Gomez-Fernandez C, Wright J, Leone JP, Larrieu R, Pegram MD. The use of neoadjuvant platinum-based chemotherapy in locally advanced breast cancer that is triple negative: retrospective analysis of 144 patients. Breast Cancer Res Treat. 2013;138(3):783-94.

31. Farmer H, McCabe N, Lord CJ, Tutt AN, Johnson DA, Richardson TB, Santarosa M, Dillon K, Hickson I, Knights C, et al. Targeting the DNA repair defect in BRCA mutant cells as a therapeutic strategy. Nature. 2005; 434(7035):917-21.

\section{Submit your next manuscript to BioMed Central and we will help you at every step:}

- We accept pre-submission inquiries

- Our selector tool helps you to find the most relevant journal

- We provide round the clock customer support

- Convenient online submission

- Thorough peer review

- Inclusion in PubMed and all major indexing services

- Maximum visibility for your research

Submit your manuscript at www.biomedcentral.com/submit

) Biomed Central 